\title{
Performance of Inductive Method of Model Self-Organization with Incomplete Model and Noisy Data*
}

\author{
Natalia Ponomareva, ${ }^{1,2}$ Mikhail Alexandrov, ${ }^{3,4}$ Alexander Gelbukh ${ }^{5,4}$ \\ ${ }^{1}$ University of Wolverhampton, $U K$ \\ ${ }^{2}$ Polytechnic University of Valencia, Spain \\ ${ }^{3}$ Autonomous University of Barcelona, Spain \\ ${ }^{4}$ Social Networks Research Center, University of Central Europe in Skalica, Slovakia \\ ${ }^{5}$ Center for Computing Research, National Polytechnic Institute, Mexico \\ \{nponomareva,dyner1950\}@mail.ru,www.gelbukh.com
}

\begin{abstract}
Inductive method of model self-organization (IMMSO) developed in 80s by A. Ivakhnenko is an evolutionary machine learning algorithm, which allows selecting a model of optimal complexity that describes or explains a limited number of observation data when any a priori information is absent or is highly insufficient. In this paper, we study the performance of IMMSO to reveal a model in a given class with different volumes of data, contributions of unaccounted components, and levels of noise. As a simple case study, we consider artificial observation data: the sum of a quadratic parabola and cosine; model class under consideration is a polynomial series. The results are interpreted in the terms of signal-noise ratio.
\end{abstract}

\section{Introduction}

\subsection{Inductive method of model self-organization}

We study the Inductive Method of Model SelfOrganization (IMMSO), an evolutionary machinelearning (ML) algorithm that has important applications in Data Mining and other ML tasks. This method aims to determine the optimal complexity of a model that could describe or explain a given set of experimental observation data. Note that the method determines the complexity of the model and not the specific values of its parameters.

\footnotetext{
* Work done under partial support of Mexican Government (SNI, CONACYT, SIP IPN, COTEPABE IPN) for the third author.
}

By a model we mean a formula, equation, algorithm, etc. Since IMMSO was originally developed in the Soviet Union and there is insufficient literature available on it in English, we will briefly describe the method and the conditions of its application.

IMMSO was suggested and developed by Ivakhnenko et al. in 80 s [2, 4, 9]. This method does not require any a priori information on distribution of parameters of the objects under consideration. The method is very efficient in Data Mining problems under these circumstances. However, if a priori information is available, then it can be outperformed by other methods, such as some Pattern Recognition methods, which can give significantly better results.

The method has one restriction: it is not applicable to a continuous class of models, because it is based on the competition of models. This is why this method is called inductive. Its main principle of model selection is the principle of stability: the models describing different subsets of a given data set must be similar.

Here is how the IMMSO works:

1. An expert defines a sequence of models, ordered from the simplest to more complex ones.

2. Experimental data are randomly divided into two data sets: training data and control data.

3. For a given kind of model, the best parameters are determined using first the training data and then the control data set. For this, an internal criterion of concordance between the model and the data can be used, e.g., the least squares criterion.

4. Both models are compared on the basis of an external criterion, such as the criterion of regularity, criterion of unbiasedness, etc. If this external criterion, or a combination of such 
criteria, achieves a stable optimum, the process is finished. Otherwise, more complex model is considered and the process is repeated from the step 3.

The method uses the notions of internal criteria and external criteria. The difference is that internal criteria use the same data both to determine the model parameters and to evaluate its quality, while external ones use different data for this purpose. In practice, usually external criteria are constructed as nonnegative functions with zero being the optimum.

The external criterion can reach an optimum when the data contain:

- a regular component defined by the chosen model structure, and

- a random component: noise.

Indeed, a too simple model ignores the noise, but at the same time it does not recognize the regularities in the data. On the other hand, a too complex model can reflect very well such regularities but also will be too sensitive to noise. In both cases, the differences between two models will be significant and the values of the penalty function-external criterion, large. The principle of model self-organization consists in that an external criterion passes through its optimum as the model complexity is gradually increased. It has been proved that for noisy data and a small data sample, the minimum of mathematical expectation of an external criterion is unique [4].

However, if a chosen class of models does not reflect the regularities in the data or reflects them only partially then the "random" component in the data is not exactly noise but rather an undefined, or unaccounted for, component. The contribution of such a component to experimental data can be comparative with the contribution of the model component. This distorts the results and the minimum found by the algorithm may be located far from the real best point. This is why it is very important for the user to correctly choose the model class. This choice should be based on the knowledge of the nature of the objects described by the data. If there is no information about this nature, some general class of models that is guaranteed to include the real model class is chosen.

IMMSO was successfully used in numerous applications in science and engineering for identification of physical laws, approximation of multidimensional processes, and short-term and longterm forecasting of processes and events [2, 9]. For example, it was used in geology [3], applied mathematics $[6,13]$, and text processing $[1,10,12]$. A review of various variants of IMMSO can be found in [5]. Further development of inductive modeling is considered in $[7,8]$.

\subsection{Limitations of the method}

The IMMSO can give unsatisfactory results if:

1. The data volume is very limited

2. The chosen model class essentially differs from the real model underlying the data's nature

3. The level of noise is too high

In more detail, we can comment these conditions as follows:

1. Data volume. Obviously, the limited data set does not allow revealing highly complex models. If model complexity is associated with the number of its parameters then the number of data points can not be less than the number of model parameters. In practice, this condition is much more rigid; see Section 2.1.

2. Model class. If the selected model class does not reflect the real structure of the objects being modeled, then the results are unpredictable. Usually, in this case oversimplified models are suggested by the method, due to the principle of stability - a consequence of the criterion of regularity; see Section 2.3.

3. Noise level. If the level of external noise is too high then the results depend on a specific measurement. In this case, again, usually oversimplified models are suggested by the method due to the principle of independence from data - a consequence of the criterion of unbiasedness; see Section 2.3.

\subsection{Research questions}

Noise immunity of inductive modeling was considered in detail in [2, 4], and other works related with Neural Network training [8]. They concern mainly the influence of white noise. In this work, we study inductive modeling in presence of unaccounted components in addition to the noise.

As case study, we consider polynomial functions. Obviously, the results may vary if another class of functions is considered, for example, Fourier series, etc., or with another type of models, such as differential models. However, we believe that our qualitative conclusions will still hold. 
The paper is structured as follows: Section 2 describes our experimental settings and presents the definitions of the external criterion. Section 3 presents the obtained results. Section 4 gives the conclusions.

\section{Experimental settings}

\subsection{Model class}

Let us first define the notions of model, components of model, model complexity, and noise. The notion of a model in natural and technical sciences implies the following:

- Mathematical model represented by an equation or a system of equations

- Algorithmic model represented by a list of rules for data transformation

In this paper, mathematical models are considered in the following form:

$$
\psi(x)=F(x)+\varphi(x)+\omega(x)
$$

where $x$ is an independent variable defining the data observations points; $F(x)$ is a numerical function of a given class reflecting principal components of the model; $\varphi(x)$ is a numerical function reflecting unaccounted for-additional-components of the model, and $\omega(x)$ is a random component-noise.

As a case study, we assume the following classes of functions:

- Principal components correspond to the members of a polynomial: $\quad F(x)=a_{0}+a_{1} x+a_{2} x^{2}+\ldots$, where $a_{0}, a_{1}, a_{2}$, etc. are unknown coefficients.

- Unaccounted for component is a periodical function $\varphi(x)=b \cos (k x)$, where $b$ is a parameter defining its contribution to the observation data; $k$ defines variability of the additional component, which can be close or far from the variability of the principal components.

- Noise $\omega(x)$ is a random function with zero mean value and with variance $s^{2}$.

The models (1) are used in time series analysis related with seasonal changes of temperature. These models have been considered in [2].

Note that given the coefficient $k \geq 5 \pi$, the period of harmonic function is $T \leq 2 \pi / 5 \pi=0.4$. It is easy to see that such a period allows the function to be defined on the interval $[-1,1]$ at least 5 times.

When applying the IMMSO, we suppose that:
- Additional component $\varphi(x)$ is unknown

- Variance of noise is unknown

The IMMSO implies determining the best polynomial in the form

$$
F(x)=a_{0}+a_{1} x+a_{2} x^{2}+\ldots
$$

It is well-known that the method of least squares, which is usually used in IMMSO, decreases the effect of noise $\sqrt{n}$ times when the number of data points exceeds the number of parameters of the model $n$ times. Therefore, in order to decrease the effect of noise 2 or 3 times, the number of observations should exceed the number of parameters $5-10$ times. This is what is usually recommended in practice by the IMMSO experts and developers [2].

With this, to study the effect of noise with a small number of data points, one needs to make several measurements of the noise component and then generalize the results.

The term model complexity usually denotes the number of components of the model and the strength of relations between them. In case of polynomial function, the model complexity can be defined as the highest power of the polynomial.

\subsection{Observation data}

The observation data we used for our experiments are the values of function $G(x)=x^{2}+b \cos (5 \pi x)$ calculated at $N$ points from the interval $[-1,1]$, where $x^{2}$ is the principal component of the model and $b \cos (5 \pi x)$ as the additional component. In our experiments, we consider the following values: $b \in\{0.1,0.2,0.5\}$, i.e., $10 \%, 20 \%$, and $50 \%$ of the maximum value for the principal component $x^{2}$ on the interval $[-1,1]$, respectively.

We added to the data some noise: normally distributed random values with zero mean and rootmean-square value $s \in\{0.1,0.2,0.5\}$. It also gives $10 \%, 20 \%$, and $50 \%$ of the maximum value for $x^{2}$ on the interval $[-1,1]$.

The number of points is $N \in\{50,1000\}$. Therefore the training set and the control set contain 25 and 500 points, respectively. Consider a complete polynomial (2) of the fourth order. It contains 5 members. In this case, each polynomial parameter is "covered" by 5 and 100 points, respectively. This decreases the error of noise $\sqrt{5} \approx 2$ and $\sqrt{100}=10$ times, respectively. In case of small number of points $(N=50)$ and high level of noise $(s=50 \%)$, we implemented 5 realizations of the noise component and found the solution for each realization. 
Table 1. Energetic ratios between model components

\begin{tabular}{lcccccccccc}
\hline Ratio & $b / s$ & $0.1 / 0.1$ & $0.1 / 0.2$ & $0.1 / 0.5$ & $0.2 / 0.1$ & $0.2 / 0.2$ & $0.2 / 0.5$ & $0.5 / 0.1$ & $0.5 / 0.2$ & $0.5 / 0.5$ \\
\hline$W_{\varphi} / W_{F}$ & 0.025 & 0.025 & 0.025 & 0.10 & 0.10 & 0.10 & 0.625 & 0.625 & 0.625 \\
$s^{2} / W_{F}$ & 0.05 & 0.20 & 1.25 & 0.05 & 0.20 & 1.25 & 0.05 & 0.20 & 1.25 \\
\hline
\end{tabular}

\subsection{Evaluation of results and energetic ratios}

For application of the IMMSO, the following complete polynomial models are considered:

$$
\begin{aligned}
& F_{0}(x)=a_{0} \\
& F_{1}(x)=a_{0}+a_{1} x \\
& F_{2}(x)=a_{0}+a_{1} x+a_{2} x^{2} \\
& F_{3}(x)=a_{0}+a_{1} x+a_{2} x^{2}+a_{3} x^{3}, \text { etc. }
\end{aligned}
$$

We evaluate correctness of a solution as coincidence of orders of polynomial models. That is, if the second order polynomial is revealed (independently of the number of its members) then the method is considered to give a correct result. Note that the specific values of polynomial coefficients do not matter: we are only interested in the optimal complexity of the model, not in the specific optimal values of its parameters.

If the coefficient of the highest polynomial member is significantly less than the coefficient of the next member - say, by 2 orders of magnitude - then the model complexity should be decreased. For example, the following third order polynomial $F(x)=0.3+$ $0.1 x+7 x^{2}+0.05 x^{3}$ on the interval $[-1,1]$ has in effect second order. However, all such cases proposed by the method can be considered to make a decision in practice. In any case, such a solution should be justified, taking into account, for example, the level of noise, etc.

Obviously, our function in the form of a polynomial function, periodical component, and noise is a particular case of empirical models that could be treated with IMMSO. In order to generalize the results, one should describe the models considering energetic ratios.

The power and the root-mean-square value of any function $f(x)$ defined on a given interval $T$ is given by

$$
W=\frac{1}{T}\left[\int_{T} f^{2}(x) d x\right] .
$$

For the functions $F(x)=x^{2}$ and $\varphi(x)=b \cos (k x)$ we have, respectively,

$$
W_{F}=1 / 5, W_{\varphi}=b^{2} / 2 .
$$

Table 1 shows the ratios $\eta=W_{\varphi} / W_{F}$ and $\varepsilon=s^{2} / W_{F}$ for the selected values of amplitude $b$ (additional component) and selected values of root-mean-square $s$ (noise).

It is easy to see that our experimental conditions are rather complex: the ratio of additional to principal components varies from some $3 \%$ to some $60 \%$, and the ratio of noise to principal component varies from $5 \%$ to $125 \%$

\subsection{Methods and criteria}

In our experiments, the IMMSO was to find a polynomial function of a given form (2). The observation data (artificial data) were divided into training and control sets, the experiments were conducted, and the external criteria were calculated. The winner was defined on the basis of minimum of one or several external criteria described below.

The models, which were constructed at each step of the IMMSO algorithm, used the Least Squares Method (LSM) [11]. Recall that we have two separate models: the first one based only on the training data set, and the second one based only on the control data set. In constructing these models, LSM minimizes the variance of error between the model predictions and the observation data:

$$
\varepsilon^{2}=\|F-D\|,
$$

where $F=\left\{F_{i}\right\}$ is the vector of model function values at the points of observations $\left\{x_{i}\right\}$ and $D=\left\{D_{i}\right\}$ is the vector of the observation data.

There are many variants of external criteria. Usually, in applications of the IMMSO the following two criteria are considered:

- criterion of regularity $K_{r}$

- criterion of unbiasedness $K_{u}$

Both criteria use the training data set and the test data set. The criterion of regularity reflects the difference between the model data and the control data when the model is constructed on the basis of training data set. So, this criterion evaluates the stability of the model with respect to data variation. The criterion of unbiasedness reflects the difference between the two models - those constructed on the training set and on the control set, respectively. So, this criterion evaluates independence of the model from the data. 
Table 2. Values of combined criterion for $N=50$

\begin{tabular}{lcccccc}
\hline Realization of noise & $F_{0}(x)$ & $F_{1}(x)$ & $F_{2}(x)$ & $F_{3}(x)$ & $F_{4}(x)$ & $F_{5}(x)$ \\
\hline Noise 1 & 0.6540 & 0.7989 & 0.967 & 0.9694 & 1.0629 & 1.0612 \\
Noise 2 & 0.7040 & 0.7522 & 0.7219 & 0.7312 & 0.8234 & 0.7954 \\
Noise 3 & 1.0774 & 1.1167 & 1.0193 & 1.1010 & 1.1014 & 1.1344 \\
Noise 4 & 0.4885 & 0.6407 & 0.5565 & 0.5655 & 0.5473 & 0.5049 \\
Noise 5 & 0.6419 & 0.6166 & 0.5117 & 0.5659 & 0.6545 & 0.9778 \\
\hline Average & 0.71316 & 0.78502 & 0.75528 & 0.7866 & 0.8379 & 0.89474
\end{tabular}

Table 3. Values of combined criterion for $N=1000$

\begin{tabular}{lcccccc}
\hline Realization of noise & $F_{0}(x)$ & $F_{1}(x)$ & $F_{2}(x)$ & $F_{3}(x)$ & $F_{4}(x)$ & $F_{5}(x)$ \\
\hline Noise 1 & 0.5855 & 0.5852 & 0.4833 & 0.4833 & 0.4842 & 0.4845 \\
Noise 2 & 0.5790 & 0.5858 & 0.5026 & 0.5033 & 0.5051 & 0.5056 \\
Noise 3 & 0.5754 & 0.5973 & 0.5346 & 0.5353 & 0.5353 & 0.5397 \\
Noise 4 & 0.5853 & 0.5866 & 0.5238 & 0.5394 & 0.5433 & 0.5444 \\
Noise 5 & 0.5774 & 0.5817 & 0.5416 & 0.5440 & 0.5429 & 0.5433 \\
\hline Average & 0.5805 & 0.5873 & 0.5172 & 0.5211 & 0.5222 & 0.5235
\end{tabular}

Different forms of these criteria can be proposed. A specific form depends on a specific given problem. In our case, we use these criteria in the following forms:

$$
K_{r}=\frac{\sqrt{\sum_{C}\left(q_{i}(T)-q_{i}\right)^{2}}}{\sqrt{\sum_{C} q_{i}^{2}}}, K_{u}=\frac{\sqrt{\sum_{T+C}\left(q_{i}(T)-q_{i}(C)\right)^{2}}}{\sqrt{\sum_{T+C} q_{i}^{2}}},
$$

where $T$ and $C$ are the systems of equations (2) used for training and control, respectively; $q_{i}(T)$ and $q_{i}(C)$ are the model data that is the right part of the equations with the parameters determined on the training and control set, respectively; $q_{i}$ are the experimental (artificial) data, i.e., the left part of the equations; $i$ is the number of the equation.

Sometimes a model can be better than another one according to the first criterion but worse according to the second one. A combined criterion can be used, e.g.:

$$
K=\lambda K_{r}+(1-\lambda) K_{u},
$$

where $\lambda$ is a user-defined coefficient of preference. In our experiments, we used $\lambda=2 / 3$, i.e., we consider the criterion of regularity as more important.

\section{Results of inductive modeling}

\subsection{Sensitivity to the volume of data}

We used the following data in this experiment:

- $\quad$ amplitude of the additional component $b=0.1$ (the lowest level)

- level of noise $s=0.5$ (the highest)
For this case, we have the relations $\eta=2.5 \%$ and $\varepsilon=125 \%$.

We considered 5 realization of noise. Tables 2 and 3 show the values of combined criterion (6). In graphical form this is shown in Fig. 1.

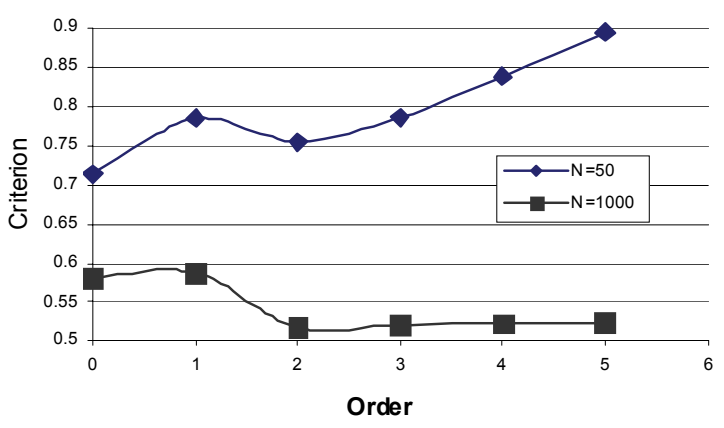

Fig. 1 Dependence on volume of data

One can see that the realizations significantly differ from each other. The average value reaches its minimum for the 0 -order model. However, in 2 cases of 5 , the winner is the second order model. This means that the result was correct in $40 \%$ of our test runs.

In all cases, the minimum is reached for the second order model, due to strong filtration.

\subsection{Sensitivity to inexactness of model}

We used the following data in this experiment:

- $\quad$ total number of points $N=50$

- level of noise $s=0.1$ (the lowest) 
Table 4. Average values of combined criterion for $b \in\{0.1,0.2,0.5\}$

\begin{tabular}{|c|c|c|c|c|c|c|}
\hline Additional component & $F_{0}(x)$ & $F_{1}(x)$ & $F_{2}(x)$ & $F_{3}(x)$ & $F_{4}(x)$ & $F_{5}(x)$ \\
\hline$b=0.1 \quad(\eta=2.5 \%)$ & 0.4727 & 0.4954 & 0.2030 & 0.2162 & 0.2200 & 0.2271 \\
\hline$b=0.2 \quad(\eta=10 \%)$ & 0.5061 & 0.5341 & 0.2760 & 0.2877 & 0.2903 & 0.2942 \\
\hline$b=0.5 \quad(\eta=62.5 \%)$ & 0.5646 & 0.5935 & 0.4236 & 0.4319 & 0.4207 & 0.4241 \\
\hline
\end{tabular}

Table 5. Spectrum of $x^{2}$

\begin{tabular}{lcccccc}
\hline Harmonics & const & $\cos (\pi x)$ & $\cos (2 \pi x)$ & $\cos (3 \pi x)$ & $\cos (4 \pi x)$ & $\cos (5 \pi x)$ \\
\hline Value & 0.223 & 0.084 & 0.005 & 0 & 0 & 0 \\
\hline
\end{tabular}

Table 6. Average values of combined criterion for $s \in\{0.1,0.2,0.5\}$

\begin{tabular}{llcccccc}
\hline Noise & & $F_{0}(x)$ & $F_{1}(x)$ & $F_{2}(x)$ & $F_{3}(x)$ & $F_{4}(x)$ & $F_{5}(x)$ \\
\hline$s=0.1$ & $(\varepsilon=5 \%)$ & 0.4847 & 0.5199 & 0.2027 & 0.2080 & 0.2103 & 0.2131 \\
$s=0.2$ & $(\varepsilon=20 \%)$ & 0.5489 & 0.5955 & 0.3387 & 0.3483 & 0.3711 & 0.3827 \\
$s=0.5$ & $(\varepsilon=125 \%)$ & 0.6439 & 0.7070 & 0.6134 & 0.6421 & 0.6535 & 0.6640 \\
\hline
\end{tabular}

For this case, we have $\varepsilon=5 \%$. In this experiment we considered five realizations of noise for each value of $b$. Table 4 contains the average values of combined criterion (6). The results are presented in graphical form in Fig. 2.

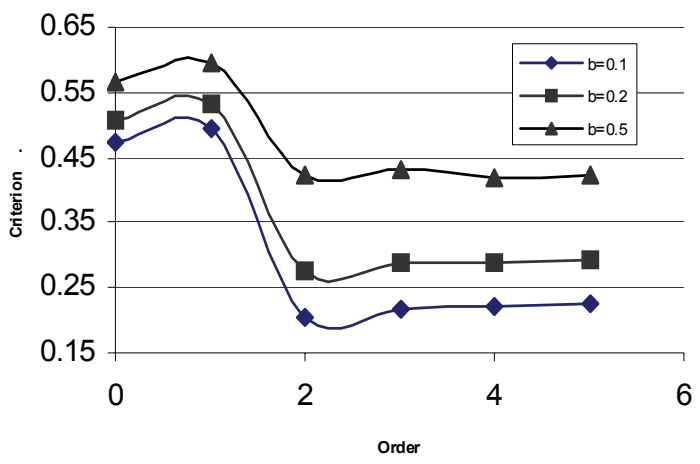

Fig. 2. Dependence on the unaccounted for function

The best model was the one of second order for all values of $b$. This model was the best for 12 realizations out of 15 ; in the rest of the cases the winner was the third order model. Therefore, we obtained the correct result in $80 \%$ of cases.

It should say that the variability of principal and unaccounted for components, i.e., $x^{2}$ and $b \cos (5 \pi x)$, is significantly different. Table 5 presents the spectrum of $x^{2}$ on the interval $[-1,1]$.

It is easy to see that since second harmonic the spectrum of the principal component is practically zero. The additional component has also fifth harmonic $\cos (5 \pi x)$. Therefore, both components are orthogonal. This is why the obtained results are rather good. However, this issue needs especial attention.

\subsection{Sensitivity to noise}

We used the following data in this experiment:

- $\quad$ total number of points $N=50$

- amplitude of the additional component $b=0.1$ (the lowest)

For this case we have $\eta=2.5 \%$. We considered three realizations for each level of noise. Table 6 shows the average values of the combined criterion (6). These results are presented in graphical form in Fig. 3.

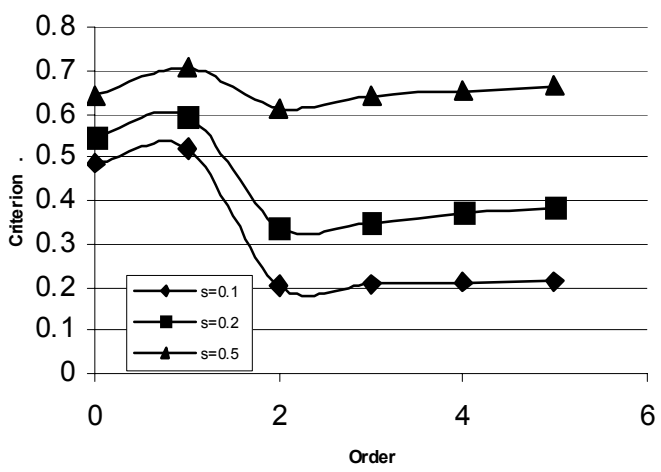

Fig. 3. Dependence on noise

The winner was the second order model for all values of $s$. With this, for $s=0.1$ all realizations provided the correct result; for $s=0.2$, we obtained two incorrect results out of five; for $s=0.5$, two incorrect results out of five as well. 
Table 7. Values of all criterion for the case $b=s=0.1$

\begin{tabular}{lccccccc}
\hline Criterions & & $F_{0}(x)$ & $F_{I}(x)$ & $F_{2}(x)$ & $F_{3}(x)$ & $F_{4}(x)$ & $F_{5}(x)$ \\
Regularity & $K_{r}$ & 0.6945 & 0.7002 & 0.2299 & 0.2301 & 0.2374 & 0.2374 \\
Unbiasedness & $K_{u}$ & 0.0367 & 0.1102 & 0.0760 & 0.0849 & 0.1094 & 0.1158 \\
Combined criterion & $K$ & 0.4752 & 0.5035 & 0.1786 & 0.1817 & 0.1947 & 0.1969 \\
\hline
\end{tabular}

Table 8. Values of all criterion for the case $b=s=0.5$

\begin{tabular}{lccccccc}
\hline Criterions & & $F_{0}(x)$ & $F_{I}(x)$ & $F_{2}(x)$ & $F_{3}(x)$ & $F_{4}(x)$ & $F_{5}(x)$ \\
Regularity & $K_{r}$ & 0.91954 & 0.93776 & 0.79044 & 0.8038 & 0.80218 & 0.79534 \\
Unbiasedness & $K_{u}$ & 0.15672 & 0.28392 & 0.31076 & 0.3478 & 0.41604 & 0.44244 \\
Combined criterion & $K$ & 0.66526 & 0.71982 & 0.63054 & 0.6518 & 0.67348 & 0.67770 \\
\hline
\end{tabular}

\subsection{Model self-organization with different criteria}

In this experiment, we used the minimum number of points $N=50$.

1. The best case for modeling:

- amplitude of additional component $b=0.1$ (the lowest)

- level of noise $s=0.1$ (the lowest)

For this case we have $\eta=2.5 \%$ and $\varepsilon=5 \%$. The results are presented in Table 7 and in Fig. 4.

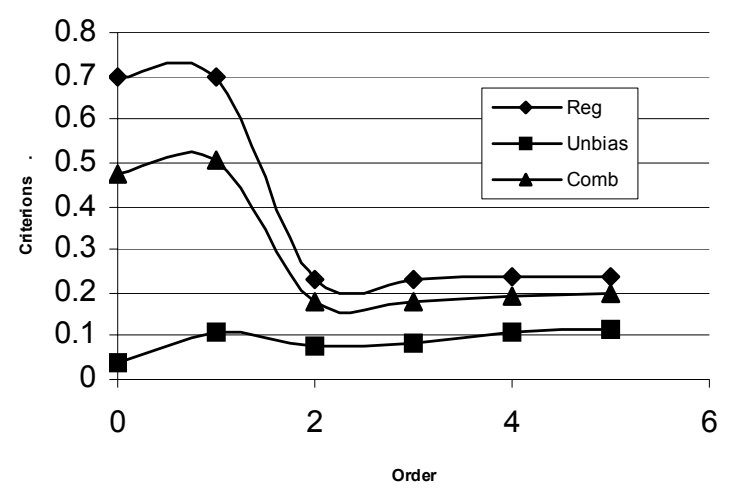

Fig. 4. Criteria for the best case

One can see that the criterion of regularity gives the correct result: the second order model. The criterion of unbiasedness gives the best result for 0 -order model, with a local minimum is at the second order model. The combined criterion reaches its minimum on the correct second order model.

2. The worst case for modeling:

- $\quad$ amplitude of the additional component $b=0.5$ (the highest)

- level of noise $s=0.5$ (the highest)
For this case we have $\eta=62.5 \%$ and $\varepsilon=125 \%$. The results are presented in Table 8 and in Fig. 5.

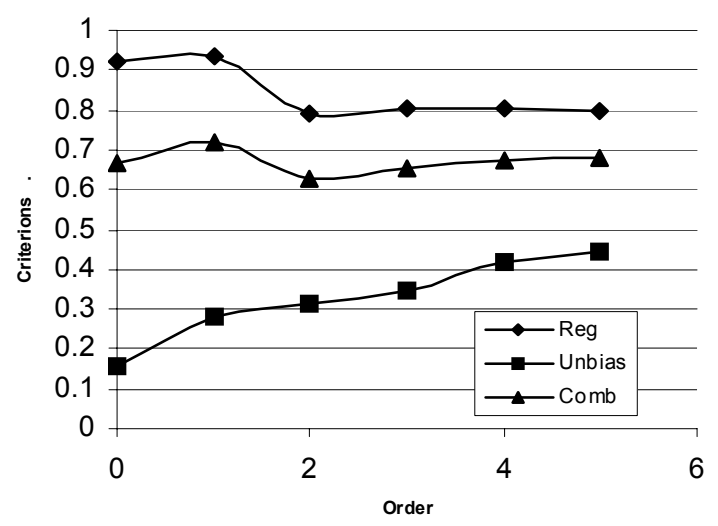

Fig. 5. Criteria for the worst case

One can see that the criterion of regularity gives the correct result: second order model. The criterion of unbiasedness gives the best result for 0 -order model. The combined criterion reaches its minimum on the correct second order model, though it is rather smooth and does not distinguish the models well.

\section{Conclusions}

We studied the performance of the inductive method for the test case of polynomial model with unaccounted for trigonometric function and some noise. In general, the method demonstrated low sensitivity to noise. The results depend on the ratios of the principal component, the additional components, and the noise.

Usually, the works studying noise sensitivity for Ivakhnenko's inductive method consider normal white noise. In the paper, we considered another case, when this distortion is created by unaccounted for model components in addition to the usual noise. 
We have presented the results of inductive modeling for different volumes of data, and contributions of unaccounted for model component and the level of noise. Such results can be easily generalized to take into account the ratios of principal component, additional component, and noise.

In the future, we plan to use spectral approach to analyze the noise sensitivity of inductive modeling.

\section{References}

[1] Alexandrov, M., Blanco, X., Ponomareva, N., Rosso, P. (2007): Constructing Empirical Models for Automatic Dialog Parameterization. In: Text, Speech, Dialog, TSD 2007. Springer, LNCS 4629, 455-462.

[2] Ivakhnenko, A. (1982): Inductive Method of Model SelfOrganization of Complex Systems, Kiev, Tekhnika Publ. (in Russian).

[3] Ivakhnenko, A., Peka, G., Vostrov, N. (1984): Combined Method for Modeling Petroleum and Water Fields. Kiev, Naukova Dumka Publ. (in Russian).

[4] Ivakhnenko, A., Stepashko, V. (1985): Noise Immunity of Modeling, Naukova Dumka Publ. (in Russian).

[5] Ivakhnenko, A., Ivakhnenko, G. (1995): The Review of Problems Solvable by Algorithms of the Group Method of Data Handling (GMDH). Pattern Recognition and Image Analysis, Vol. 5, No. 4, 527-535.

[6] Ivakhnenko, A., Ivakhnenko, G. (1995): Simplified Linear Programming Algorithm as Basic Tool for Open-
Loop Control. System Analysis Modeling Simulation (SAMS), vol. 12, No. 1, 11-18.

[7] Ivakhnenko, A., Ivakhnenko, G. (2000): Problems of Further Development of the Group Method of Data Handling Algorithms.Part I. Pattern Recognition and Image Analysis, vol. 10, No. 2, 187-194

[8] Ivakhnenko, A., Ivakhnenko, G., Savchenko, E., Wunsch, D. (2002): Problems of Further Development of GMDH Algorithms: Part 2. Pattern Recognition and Image Analysis, Vol. 12, No. 1, 6-18.

[9] Madala, H., Ivakhnenko A. (1994): Inductive Learning Algorithms for Complex System Modeling. CRC Press.

[10] Makagonov, P., Alexandrov, M. (2002): Constructing empirical formulas for testing word similarity by the inductive method of model self-organization. In: Advances in Natural Language Processing. Springer, LNAI 2389, 239247.

[11] Shoup, T. (1982): Solution of engineering problems with computers.

[12] Sidorov, G. (2005): Linguist's Intelleigent Workplace for Spanish Language. In: Advances in artificial intelligence and computer science, J. Research on computing science, vol. 14, pp. 173-178.

[13] Triseev, Yu. P. (1987): Approaches to the Solution of Mathematical Programming Problems on the Basis of Heuristic Self-Organization. Automation and Information Sciences, vol. 20, No. 2, 30-37 (in Russian). 